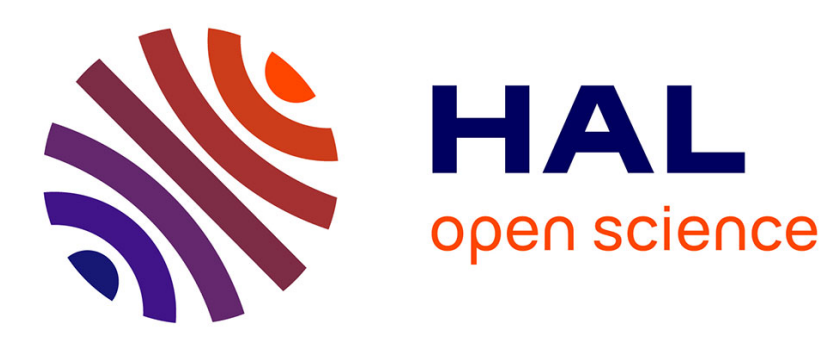

\title{
Convection of local level set function for moving surfaces and interfaces in forming flow \\ Thierry Coupez
}

\section{To cite this version:}

Thierry Coupez. Convection of local level set function for moving surfaces and interfaces in forming flow. Materials Processing and Design, Modeling, Simulation and Applications, NUMIFORM '07: 9th International Conference on Numerical Methods in Industrial Forming Processes, Jun 2007, Porto, Portugal. p. 61-66, 10.1063/1.2740790 . hal-00510556

\section{HAL Id: hal-00510556 \\ https: / hal-mines-paristech.archives-ouvertes.fr/hal-00510556}

Submitted on 4 Apr 2011

HAL is a multi-disciplinary open access archive for the deposit and dissemination of scientific research documents, whether they are published or not. The documents may come from teaching and research institutions in France or abroad, or from public or private research centers.
L'archive ouverte pluridisciplinaire HAL, est destinée au dépôt et à la diffusion de documents scientifiques de niveau recherche, publiés ou non, émanant des établissements d'enseignement et de recherche français ou étrangers, des laboratoires publics ou privés. 


\title{
Convection Of Local Level Set Function For Moving Surfaces And Interfaces In Forming Flow
}

\author{
Thierry Coupez \\ CEMEF-ENSMP-umr CNRS 7635 BP 207 - 06904 Sophia Antipolis Cedex, France \\ e-mail:Thierry.Coupez@cemef.cma.fr
}

\begin{abstract}
We propose to improve the Level Set method by introducing a convective derivative in the reinitialisation equation. Our approach allows to keep an unitary gradient for Level Set function at the interface. In this way, it is not necessary to regularize periodically the level set since the basic properties are preserved when it is convected. Moreover a local Level Set is introduced by using a sinus like function which enables to ensure a smooth transition. Examples are given in the context of finite element method for forming applications.
\end{abstract}

Keywords: Level Set, Free surface, Forming, Finite Element for Fluid flow.

PACS

\section{INTRODUCTION}

The popularity of the Level Set method is still growing since the early work of Osher [Osher 88] and its consolidation [Sethian,95]. Improvements are still going on [Chang,1996] [Peng,1999] including work on incompressible multi-phase flow problems [Sussman,1999]

In this paper we propose to improve the robustness of the method and also to simplify the implementation in case of moving free surfaces and interfaces. We will show that efficiency of Level Set method is quite improved by using this approach for very complex free surface incompressible flow problem.

The main characteristics of the proposed method are in one hand to avoid the reinitialization stage often used after different steps of convection and in other hand to truncate efficiently the Level set function outside the region of the interface without propagating instabilities.

\section{INTERFACE CAPTURING}

Let us introduce the Level Set function $\alpha$ defined in a domain $\Omega$. It gives us for each point of the domain, the distance to the interface $\Gamma$, which is defined itself by the 0 level set of point of $\alpha$.

$$
\left\{\begin{array}{l}
\alpha(x)=d(x, \Gamma), x \in \Omega \\
\Gamma=\{x \in \Omega, \alpha(x)=0\}
\end{array}\right.
$$

We focus on moving free surface or interface calculation in a general Eulerian framework. In this context, the Level Set function enables to separate the computational domain in two subdomains occupied by two different fluids. Let us assume that the fluid velocity, $v$, field is known everywhere in $\Omega$ such as the interface motion is given by the Level Set displacement solution of the following transport equation:

$$
\left\{\begin{array}{l}
\frac{d \alpha}{d t}=\frac{\partial \alpha}{\partial t}+v . \nabla \alpha=0 \\
\alpha(t=0, x)=\alpha_{0}(x)
\end{array}\right.
$$

It is well known that equation (2) does not preserve any geometric property of the Level Set function and solving this equation provides us with a function which is not anymore a distance function. Moreover the gradient of the function can become as steep as it could, while reducing considerably the advantage of the method. Indeed the interest of Level Set method is to have a regular continuous derivative function with a smooth gradient in order to help the solution of (2) by at least a first order finite element method using simple stabilization technique. It the reason why a reinitialization stage is often associated with Level Set

CP908, NUMIFORM '07, Materials Processing and Design: Modeling, Simulation and Applications edited by J. M. A. César de Sá and A. D. Santos

(C) 2007 American Institute of Physics 978-0-7354-0415-1/07/\$23.00 
usage, in order to rebuild a true Level Set function at from the solution of (2) at any time.

\section{RE-INITIALIZATION}

If is a Level Set function as defined by (1) then several interested properties can be shown and can be used to stabilize schemes:

- If $\Gamma$ is smooth enough then $|\nabla \alpha|=1$.

- If $N(\mathrm{y})$ is the normal vector of $\Gamma$ at point $\mathrm{y}$ : $\nabla \alpha(y)=N(y)$

Indeed, for $\tau$ small enough, $\alpha(y+\tau N(y))=s$ and thus $\nabla \alpha(y) \cdot N(y))=1$. Consequently the gradient of a Level Set function is a representation of the local normal of each level (the 0 level in particular). The underlying idea in rebuilding a Level Set is to retrieve these properties. A classical way for that is to solve the above Hamilton Jacobi equation [3].

$$
\left\{\begin{array}{l}
\frac{\partial \beta}{\partial \tau}+s(|\nabla \beta|-1)=0 \\
\beta(\tau=0, x)=\alpha(t, x)
\end{array}\right.
$$

$s$ is the sign of $\beta$ initialized by $\alpha$ and the only invariant region in this model is for $s=0$ and thus where $\alpha=$ 0 .

From the practical point of view we solve equation (3) periodically when one estimates that the Level Set function is not anymore smooth enough. It adds one more stage in the simulations.

One can notice that equation (3) is a purely convective equation. Indeed, we can rewrite the gradient term by $|\nabla \beta|=\frac{\nabla \beta}{|\nabla \beta|} \cdot \nabla \beta \quad$ and then let introduce the convection velocity by $U=s \frac{\nabla \beta}{|\nabla \beta|}$, we get :

$$
\left\{\begin{array}{l}
\frac{\partial \beta}{\partial \tau}+U . \nabla \beta=s \\
\beta(\tau=0, x)=\alpha(t, x)
\end{array}\right.
$$

Equation (4) has the standard form of a transport equation similar to (3) and the same PDE solver can be reused. However, the underlying problem remains apparently non linear.

We use in this work a finite element method and unstructured meshes (triangles or tetrahedral). The method used for solving both (3) and (4) is a standard P1 approximation with a RFB (UPG) method for the stabilization. Note that the $\mathrm{P} 1$ approximation is essential in the Level Set since it provides a local exact interpolation when the boundary is a straight line

In (4) the fictitious time $\tau$ is related to the convective velocity $U$ of unity norm. It follows that, the fictitious time step must be of order $h$, the mesh size, indeed : $\mathrm{IU} \mid \Delta \tau=\Delta \tau=h$.

\section{CONVECTIVE RE-INITIALIZATION}

\section{One equation model}

The idea now will be to avoid the re-iniitialization stage by combining the models contain in Equation (2) and (3) in order to preserve both the interface motion and the distance property. For that we first need to link the fictitious time introduce in (3) to the physical time of (2) by :

$$
\frac{d a}{d \tau}=\frac{d a}{d t} \frac{d t}{d \tau}
$$

and let us introduce the following parameter:

$$
\lambda=\frac{d \tau}{d t}
$$

In line with the previous remark on the fictitious time step, 1 may be express as the ratio between the time step and the mesh size:

Consequently (3) may be transformed immediately taking into account the motion of the fluid domain with the help of a convective time derivative $\left(\frac{d \beta}{d t}=\frac{\partial \beta}{\partial t}+v \cdot \nabla \beta\right)$ and this is the tricky part of the rewriting. It gives us:

$$
\left\{\begin{array}{l}
\frac{\partial \alpha}{\partial t}+v \cdot \nabla \alpha+\lambda s(|\nabla \alpha|-1)=0 \\
\alpha(\tau=0, x)=\alpha_{0}(x)
\end{array}\right.
$$

The fluid flow and the interface motion is now taken into account in the following one equation model, which tends to convect strictly a distance function preserving its metric properties. 
Note that $s=s(\alpha)$ and is 1 or-1 and 0 only if $\alpha=0$. Consequently one retrieves (2) in the neighborhood of the 0 level.

The next step is to combine the convective flow velocity and the "metric" velocity U already introduced in (4) and then we obtain the simple expression:

$$
\left\{\begin{array}{l}
\frac{\partial \alpha}{\partial t}+(v+\lambda U) \cdot \nabla \alpha=\lambda s \\
\alpha(t=0, x)=\alpha_{0}(x)
\end{array}\right.
$$

And here again we get a classical convective equation that can be treated with the same PDE solver (SUPG or RFB in the context of FE).

\section{Sign definition and gradient regularization}

The sign plays a very important role in this type of equation since the numerical scheme will be introduced an upstream behavior determined by the above sign. In order to ensure a smooth transition and to force a good conservation in the neighborhood of the interface, the sign is defined by:

$$
s(\alpha)=\frac{\alpha}{|\alpha|+\varepsilon}
$$

Where $\varepsilon$ is related to the mesh size. One can use directly the following test:

$$
\begin{aligned}
& s(\alpha)=\frac{\alpha}{|\alpha|} \text { si } \alpha>\varepsilon \\
& s(\alpha)=0 \text { si } \alpha \leq \varepsilon
\end{aligned}
$$

Clearly: $\varepsilon \sim \mathrm{h}$

\section{Truncation}

Far from the interface, it is not necessary to maintain the distance function and it is common to truncate the function. Moreover the convective velocity can be also restricted to the vicinity of the interface and forced to zero elsewhere. Therefore the method focuses around the interface and does not need to work too far. However, it happens that the sudden change in gradient produces instabilities that propagate and pollute the solution. It is why we propose in the following section we generalize the Level Set technique to other kind of function. The only limitation is that these functions can be defined implicitly through a differential model. It is the case of the sinus function, as explained in the sequel.

\section{SINUSOIDAL SCALE OF LEVEL SET}

\section{1-D preview}

In fact the above Level Set model can be generalized to any kind of function having same independent measure properties. Let us take the example of a sinus function defined by:

$$
\alpha(x)=\frac{2 E}{\pi} \sin \left(\frac{\pi}{2 E} x\right) \quad ; x \in[-E, E]
$$

Extended by $\pm \frac{2 E}{\pi}$ beyond $\mathrm{E}$

$$
\alpha^{\prime}(0)=1 ; \alpha^{\prime}(\mathrm{E})=0 ; \alpha(\mathrm{E})=2 \mathrm{E} / \pi
$$

The following properties work:

And more particularly:

$$
\alpha^{\prime}=\sqrt{1-\left(\frac{\pi}{2 E} \alpha\right)^{2}}
$$

E being the thickness onto the sinus function plays a role.

This function is self determinant as solution of PDE (14). Moreover it can be generalized to any spatial dimension.

\section{Generalization}

The standard Level Set intends to maintain a unity gradient since the proposed approach the idea is to ensure a smooth continuous transition of the gradient from 1 to 0 by using a Sinus Scale Level Set method. The SS Level Set function is such as:

$$
|\nabla \alpha|=\sqrt{1-\left(\frac{\pi}{2 E} \alpha\right)^{2}}
$$

And as in (9) we replace the unity by a term depending on a itself proving: 


$$
\left\{\begin{array}{l}
\frac{\partial \alpha}{\partial t}+v \cdot \nabla \alpha+\lambda s\left(|\nabla \alpha|-\sqrt{1-\left(\frac{\pi}{2 E} \alpha\right)^{2}}\right)=0 \\
\alpha(t=0, x)=\alpha_{0}(x)
\end{array}\right.
$$

\section{Gradient regularization and final expression}

The last step will be to introduce again the metric velovity $\mathrm{U}$ in order to extract the convective part contained in (16) and to linearize properly this expression.

Let us introduce the scalar function $g$ to represent the gradient norm of $\alpha$ :

$$
g=g(\alpha)=\sqrt{1-\left(\frac{\pi}{2 E} \alpha\right)^{2}}
$$

$U=s \frac{\nabla \alpha}{|\nabla \alpha|}$ is only defined when $|\nabla \alpha| \neq 0$. It must be regularized and we propose the following expression:

$$
U_{\varepsilon}=s \frac{\nabla \alpha}{\varepsilon+(1-\varepsilon)|\nabla \alpha|}
$$

In that case $\left|U_{\varepsilon}\right|=\frac{|\nabla \alpha|}{\varepsilon+(1-\varepsilon)|\nabla \alpha|}$ is not anymore exactly 1 far from the interface and (18) must be slightly modified to account for it:

$$
g_{\varepsilon}=\frac{|\nabla \alpha|}{\varepsilon+(1-\varepsilon)|\nabla \alpha|} g
$$

and finally the final model we propose take the following linearized form:

$$
\left\{\begin{array}{l}
\frac{\partial \alpha}{\partial t}+\left(v+\lambda U_{\varepsilon}\right) . \nabla \alpha=\lambda s g_{\varepsilon} \\
\alpha(t=0, x)=\alpha_{0}(x)
\end{array}\right.
$$

\section{TIME DISCRETIZATION}

We choose a second order time stepping scheme and by introducing $u=v+\lambda U_{\varepsilon}$ and $f=\lambda s g_{\varepsilon}$ problem (20) takes the general form:

$$
\left\{\begin{array}{l}
\frac{\partial \alpha}{\partial t}+u . \nabla \alpha=f \\
\alpha(0, x)=\alpha_{0}(x)
\end{array}\right.
$$

This equation is integrated in time by using the following second order scheme:

$$
\frac{1}{\Delta t} \alpha_{t}+u . \nabla \alpha_{t}=f \frac{1}{\Delta t} \alpha_{t-\Delta t}+u . \nabla \alpha_{t-\Delta t}
$$

The sp

atial approximation is achieved by using a Finite Element method. The necessary upwind required by such a type of equation is performed by a SUPG like method. Let us introduce $\mathrm{K}(\Omega)$ a mesh of $\Omega$ of simplex elements (triangles, tetrahedra). We choose the simplest approximation space made of piecewise linear elements.

$A_{h}=\left\{a_{h} \in C(\Omega),\left.\quad a_{h}\right|_{\kappa} \in P^{1}(\kappa), \forall \kappa \in \mathrm{K}(\Omega)\right\}$

$C(\Omega)$ and $P^{1}(\kappa)$, denoting respectively the continuous function on $\Omega$ and the polynomial of degree 1 onto $\kappa$. One derives the test function space (Petrov Galerkin technique) from the above one by eliminating the contribution of the downstream elements in the weak formulation.

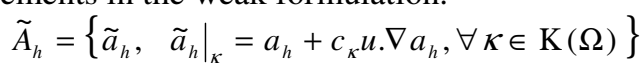

The coefficient $c_{\kappa}$ differs with the variant of the method. Here we choose to strictly upstream the scheme by choosing the following coefficient:

$$
c_{\kappa}=\frac{1}{D\left|u \cdot \nabla a_{h}\right|},
$$

D being the number of nodes per element ( 3 or 4 ).

\section{APPLICATION EXAMPLES}

Let us consider an unsteady multiphase flow problem and the proposed method to follow the moving interfaces. More precisely we are going to use the heterogeneous incompressible Navier Stokes equations to model the different fluids and interfaces in 


$$
\left\{\begin{array}{l}
\rho\left(\frac{\partial v}{\partial t}+v \cdot \nabla v\right)-\nabla \cdot(2 \eta \varepsilon(v))+\nabla p=\rho g \\
\nabla \cdot v=0 \\
v(t=0)=0 \\
\left.v\right|_{\partial \Omega}=0
\end{array}\right.
$$

In example ploted on Figure 2, there are a liquid phase, a gas phase and a solid phase. In order to separate each phase from each other, we need two Level Set function. The first one will separate the liquid and the gas, and the second one the solid and the fluid resulting from the previous mixture. Moreover, the solid is a disk initially hanged at a certain height. It drops down under its own weight.

Let introduce the Heaviside function $\mathrm{H}$ defined such as the global density and vioscosity fields are calculated by using an elementary mixture law:

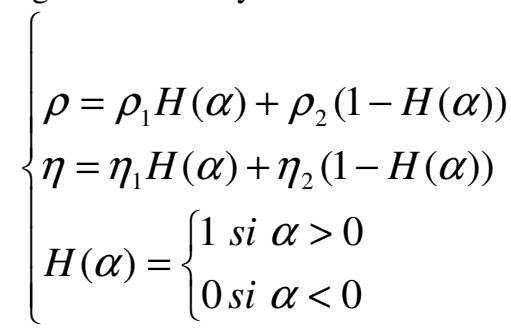

Here again a smooth transition can be managed on a certain thickness by :

$$
H_{e}(\alpha)=\left\{\begin{array}{l}
\frac{1}{2}+\frac{\alpha}{2 e} \text { si }|\alpha|<e \\
0 \text { si } \alpha<-e \\
0 \text { si } \alpha<+e
\end{array}\right.
$$

Such a regularisation can be founded also in Nagrath et al] by using the sinus function in the above definition of $H_{e}$. Note that $\alpha$ is including regularity and smoothness already and we need only to control the thickness of the mixture in the definition of $H_{e}$. From a numerical point of view this thickness cannot be independent of the mesh size. However it seems well efficient to consider a sufficient thickness to get a smooth continuous transition of rheological parameter.

Consequently the three phases problem need two functions: $\alpha_{A}$ et $\alpha_{B}$ which enable to combine by:

$$
\left\{\begin{array}{l}
\rho=\left(\rho_{1} H_{e}\left(\alpha_{A}\right)+\rho_{2}\left(1-H_{e}\left(\alpha_{A}\right)\right)\right) H_{e}\left(\alpha_{B}\right)+\rho_{3}\left(1-H_{e}\left(\alpha_{B}\right)\right) \\
\eta=\left(\eta_{1} H_{e}\left(\alpha_{A}\right)+\eta_{2}\left(1-H_{e}\left(\alpha_{A}\right)\right)\right) H_{e}\left(\alpha_{B}\right)+\eta_{3}\left(1-H_{e}\left(\alpha_{B}\right)\right)
\end{array}\right.
$$

\section{CONCLUSION}

We show in this paper that convection and reinitialisation may be totally included in a unique model equation giving rise to a new type of Level Set method. The metric properties are maintained while the conservation is still preserved in the neighbourhood of the zero level of the function. The main idea was to revisit to the Hamilton Jacobi equation used for the reinitialisation stage and to introduce the convection due to the flow in an Eulrian framework. A single constant enables to link back the fictious time classically used in this scheme to the physical time due to the convection term.

Another idea given in this paper concerns the truncature of the Level Set function beyond a certain distance from the interface. We shift to a sinus scale which can be defined also implicitly by using a differential equation. Here again a smooth transition (gradient change) greatly improves the robustness, accuracy of the method
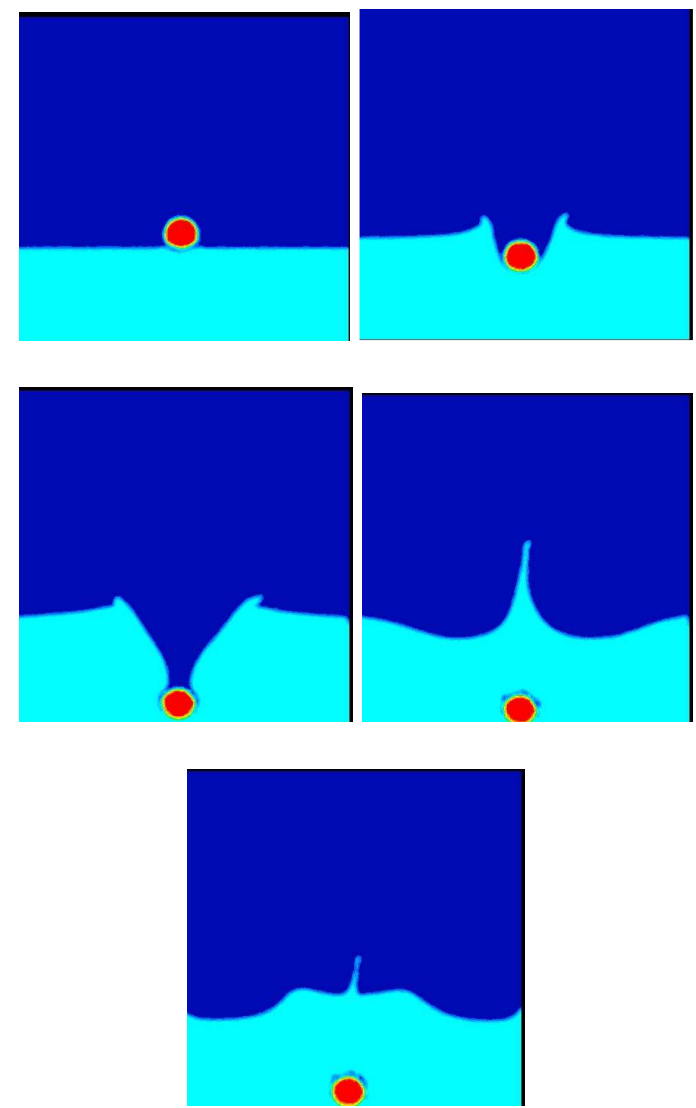

FIGURE 1. 3 phases calculation. Solid, gas, liquid. A falling sphere in a liquid. Two Level set and a global heterogeneous Navier Stokes solver. 


\section{Acknowledgements}

The author thanks the members of the Rem3Dconsortium : Atofina, Schneider Electric, Essilor, FCI, Plastic Omniuim, Transvalor , for their support

\section{References}

1. S. Osher, J.A. Sethian, "Fronts propagating with curvature dependant speed: Algorithms based on Hamilton-Jacobi formulation", J. Comput. Phys. 79,12(1988)

2. Chang,1996] Y.C. Chang, T.Y. Hou, B. Merriman, S. J. Osher, "A Level Set Formulation of Eulerian Interface Capturing Methods for Incompressible Fluid Flows", Jour. Comp. Phys., 124, pp. 449-464 (1996)

3. E. Pichelin, T. Coupez, Finite element solution of the 3d mold filling problem for viscous incompressible fluid, Comput. Methods Appl. Mech. Engrg., 163 (1998) 359—371

4. E. Bigot, T. Coupez, Capture of 3D moving free surfaces and material interfaces by mesh deformation. European Congress on Comp. Meth. in Appl. Sciences and Eng., ECCOMAS 2000, CD-Rom, Barcelona, 2000

5. S. Batkam, T. Coupez. A space-time finite element method for the thermal problem solution in $3 \mathrm{D}$ mold filling. In $5^{\text {th }}$ Int. ESAFORM conf. On Material Forming, Kraków, 2002.

6. T. Coupez, S. Marie, From a direct solver to a parallel iterative solver in $3 \mathrm{~d}$ forming simulation. Int. J. of Supercomputer Applications, 11(4):205-211, 1997.

7. T. Coupez, E. Bigot, 3D Anisotropic mesh generation and adaptation with applications. European Congress on Comp. Meth. in Appl. Sciences and Eng., ECCOMAS 2000, CD-Rom, Barcelona, 2000. 